\title{
Tunneling effects in DNA Bases Adenine and Guanine
}

\author{
Deep Kamal Kaur \\ Randhawa \\ Department of Electronics \& \\ Communication Engineering, \\ Guru Nanak Dev University \\ Regional Campus, \\ Jalandhar, India
}

\author{
Lalit M. Bharadwaj \\ Inderpreet Kaur \\ Biomolecular Electronics and \\ Nanotechnology Division (BEND), \\ Central Scientific Instruments \\ Organization (CSIO), Sector-30C, \\ Chandigarh, India.
}

\author{
M.L.Singh \\ Department of Electronics \\ Technology, \\ Guru Nanak Dev University, \\ Amritsar, \\ India.
}

\begin{abstract}
Molecular electronics has been the centre stage of research recently. Much interest has been shown by scientists in exploring the electrical and electronic properties of DNA. In this paper DNA bases Adenine and Guanine are being explored for its use as tunneling device. The molecules are applied variable bias voltage ranging from $-5 \mathrm{~V}$ to $+5 \mathrm{~V}$ to observe the flow of current using two-probe setup of Virtual Nanolab. The current-voltage characteristics obtained resemble typical tunnel diode characteristic with display of resonant tunneling phenomenon in the molecule. The two molecules Adenine and Guanine exhibit Negative Differential Resistance with a considerably large Peak to Valley Current Ratio (PVR). The observations suggest that the two molecules may be used in molecular resonant devices. The NDR characteristic suggests useful utilization of the proposed device in nanoscale digital circuits. Also the large value of PVR supports the use of Adenine in power saving highdensity integrated circuits.
\end{abstract}

Keywords: DNA bases, NDR, Tunneling, Molecular Resonant Devices.

\section{INTRODUCTION}

The scaling down of electronic devices is driving electronics into the realm of nanoelectronics. As the physical limitations of silicon are being approached due to scaling down,it is being predicted that the Moore's law will cease to exist after 2020[1].So for further miniaturization of devices some alternate materials have to be studied. For a nanoelectronic device, the element can be a carbon nanotube or a semiconducting nanowire. Molecules also provide an interesting option for use as element in nanoelectronic devices. Molecular electronic devices have the potential to offer many advantages over their silicon counterparts, including integration into much smaller areas and faster response times [2] One of the promising candidates for molecular electronics is DNA. Self-replication property of DNA advocates its suitability for creation of identical molecular electronic devices.

A molecule inserted between two electrodes acts as a quantum dot virtue its small size. As the size of molecule is very small, so its capacitance is also very small. Hence the energy $\left(\mathrm{e}^{2} / \mathrm{C}\right)$ required to add an electron to the molecule is very large. The large value of charging energy implies that the energy levels of the molecular orbitals are well separated and hence discrete in nature. The discrete nature of frontier molecular orbitals offers an excellent arrangement for utilization as Resonant Tunneling Diode (RTD). The is also supported by recent experimental reports that have shown a small group of molecules forming a nanopore that behave like a resonant tunneling diode (RTD) [3] or a device with a negative differential molecular impedance. Very recently, DNA sequencing technique has been demonstrated which is based on measurement of tunneling currents through DNA nucleotides [4].

\section{RESONANT TUNNELING DIODES}

The RTDs are well acknowledged and studied because of their potential in high speed/functionality circuits [5]. RTDs exhibit current voltage characteristics that are typical of tunnel diodes but without associated problems of large junction capacitance. Intense research has been carried out on RTDs for the last three decades for their potential use as nanoelectronic device for both analog and digital applications [6-9].

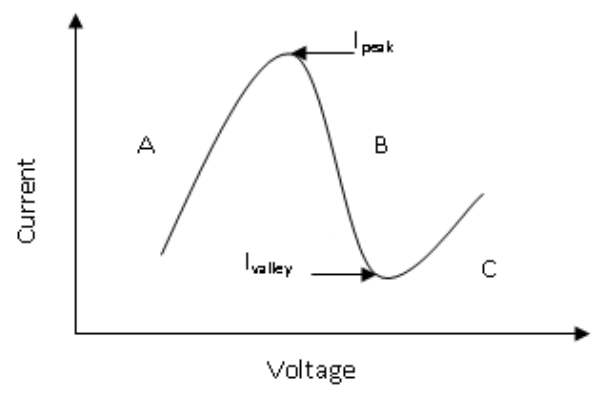

Fig. 1 Typical current-voltage characteristic of a Resonant Tunneling Diode

The operation of Resonant Tunneling Diode is based on the fact that incident energy ' $E$ ' (energy of terminal) does not match the energy 'En' of a molecular orbital. So the electrons available in the source are blocked and cannot be transmitted to the drain. As applied bias is increased, $\mathrm{E} \rightarrow \mathrm{E}_{\mathrm{n}}$ and transmission increases, reaching a maximum when $\mathrm{E}=\mathrm{E}_{\mathrm{n}}$. Rise in transmission implies rising current. This phenomenon is known as resonance and it can be considered as opening of a channel. When $E>E_{n}$, there is decreased transmission causing current flow to reduce, in effect displaying negative resistance characteristic. The typical tunneling current-voltage characteristic (as shown in Fig.1) can be divided into three sections; two positive differential resistance sections, one before peak point (section A) and other beyond the valley point (section C) with a negative differential region (section B) in between. Peak to Valley Current Ratio (PVR) is the indicator of the performance of RTD. Large PVR enables to set the distinct logic state levels and the noise tolerance of the device. High PVR is also desirable to reduce standby power consumption. Also the time elapsed by the device current to change from peak current $\left(\mathrm{I}_{\text {peak }}\right)$ and valley current $\left(\mathrm{I}_{\text {valley }}\right)$ should be small so that the device can be used for high-speed circuits [10].

DNA is the focus of research for use as nanoelectronic material. DNA consists of four DNA bases Adenine, Thymine, Guanine and Cytosine, where Adenine and Guanine are single ringed structures known as pyramidines and thymine and Cytosine are double ringed structures called purines. In this paper the pyramidines Adenine 
and Guanine are being studied to explore their current-voltage characteristics.

\section{THEORY OF ELECTRON TRANSPORT THROUGH MOLECULES}

The transport of electrons through the molecules is via the molecular orbitals. Adequate alignment of the fermi energy of the metallic contacts with the molecular orbitals of the molecules results in creation of a channel for the flow of electrons from one terminal to other via the frontier molecular orbitals of molecular island. The required alignment can be achieved by applying bias on the metallic terminals called source and drain.

A molecule is characterized by the electrons occupying the molecular orbitals where each molecular orbital is identified by unique energy value. The occupied molecular orbitals are separated from unoccupied molecular orbitals by an energy gap called HOMO-LUMO gap (HLG). When no bias is applied the fermi level of the terminals lies in the middle of the HLG. This is represented by the schematic as in Fig 2.

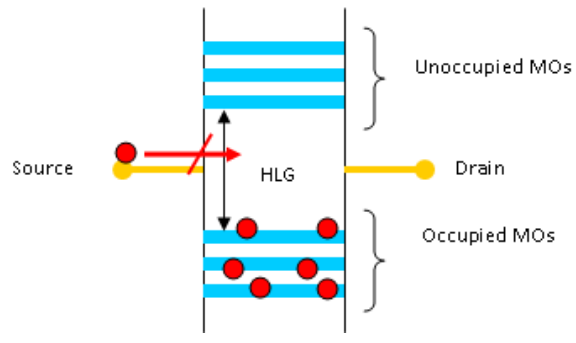

Fig 2 No conduction state

When positive voltage is applied on the source terminal the terminal is depleted of electrons. So the fermi level of source terminal is lowered while fermi level of the drain maintains its level in the middle of the HLG as shown in Fig 3. Difference in potential induces flow of electrons through the HOMO of the molecule from drain to source. The current flows from source to drain depicting p-type of behavior by the molecule. As the value of voltage is further increased, more current flows through the twoprobe setup.

When the source is applied negative potential, it increases electron population resulting in elevation of the fermi level of the source. When the energy of source is raised to the level of an unoccupied level, a channel is created for flow of electrons. The electrons flow from source to drain as illustrated in Fig 4 resulting in current flow from drain to source, representing n-type of conduction.

So it can be inferred that the electron flow will be blocked as long as the fermi energy of the electron reservoirs (source or drain) lies somewhere in the HOMO-LUMO gap. The current flows through the molecule whenever the fermi energy of the reservoirs is duly aligned with the molecular orbitals whether the orbitals are occupied or unoccupied.

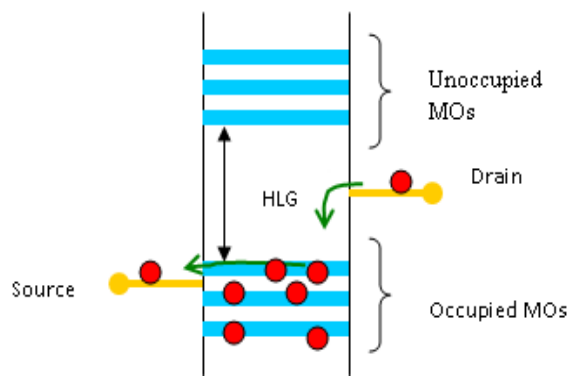

Fig 3 p-type conduction

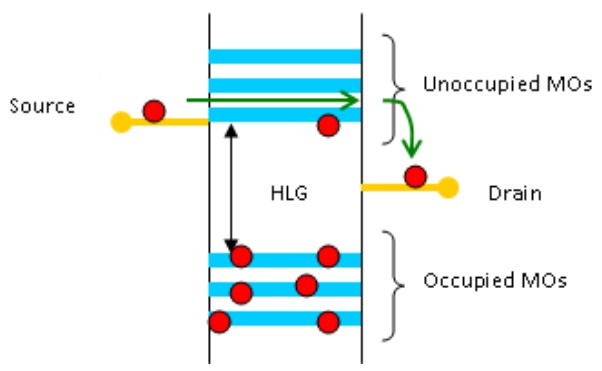

Fig 4 n-type conduction

\section{METHODOLOGY}

The focus of study in this paper is the double-ringed DNA bases Adenine and Guanine. The molecular structures of the two bases were created in HYPERCHEM 7. The backbone attached to the base molecules was removed and the bond was terminated by a hydrogen atom. The current voltage characteristics were obtained using the quantum chemical methods after inserting the molecule between two metallic terminals. The molecular structure thus obtained was simulated in the two-probe setup of Virtual Nanolab software. The current flowing through the arrangement corresponding to each voltage is calculated in ATK using Non-Equilibrium Green's Function (NEGF) combined with Density Functional Theory (DFT). Double Zeta Polarization (DZP) basis was used in these $a b$ initio electronic structure computations. Gold was used as material for the metallic terminals as it is a practical choice for designing a promising monoatomic wire.

\section{RESULTS AND DISCUSSIONS}

In this paper the two double-ringed DNA bases Adenine and Guanine are the molecules of interest. To study the electronic behavior of the molecules, the adenine and guanine molecules are inserted individually between two semi-infinite gold terminals. The metal-molecule-metal assemblies thus obtained (as in Fig 5 and Fig 7) were then simulated for current-voltage characteristics in the twoprobe setup of Virtual Nanolab software. The structures were applied a positive and negative voltage bias to obtain the currentvoltage characteristics. The characteristics are plotted with an intention to predict the electronic properties of the molecules which can be used to model the dna based electronic devices.

\subsection{Current -voltage characteristics of Adenine}

A voltage bias varying from $-5 \mathrm{~V}$ to $+5 \mathrm{~V}$ with steps of $0.1 \mathrm{~V}$ was applied at the source with drain fixed at $0 \mathrm{~V}$. The various currentvoltage values thus obtained are plotted and the curve thus obtained is shown in Fig 6. To study the V-I plot in detail the biasing of the molecule was split up into three parts; zero source voltage, positive source voltage and negative source voltage. The response for both types of polarities was then observed and the pattern of flow of current was noted.

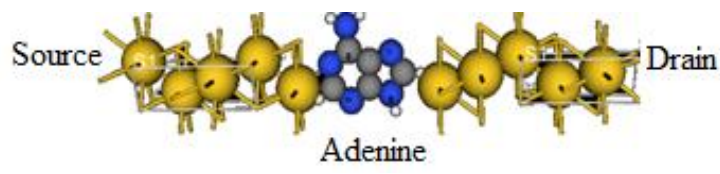

Fig 5 Adenine inserted between two Gold terminals 


\subsubsection{Zero Source Voltage}

In neutral condition the fermi level of the terminals lie in the middle of HOMO-LUMO gap $(12.76 \mathrm{eV})$ of the Adenine molecule. So that no MO is aligned with the fermi level energy of terminals, hence there is no path for flow of electrons. As the energy gap between the fermi level and LUMO is larger than room temperature thermal energy (thermal energy $=\mathrm{k}_{\mathrm{B}} \mathrm{T}=0.026 \mathrm{eV}$ at $\mathrm{T}=300 \mathrm{~K}$ ). This means that the electrons cannot jump from the terminal to the LUMO and flow toward the other terminal. So the electrons are not able to move from source to drain or vice-versa leading to zero current flows through the two-probe setup. This is analogous to the no-conduction state in the molecule.

\subsubsection{Positive Source Voltage}

As the voltage is , the current starts flowing through the twoprobe setup. The resistance offered by the molecule is $4.3 \times 10^{6} \mathrm{k} \Omega$. When applied voltage is $1.5 \mathrm{~V}$, the fermi level of the source is aligned with the HOMO (MO 35 with energy level $-6.61 \mathrm{eV}$ ) of the molecule providing an easy path for flow of electrons from drain to source through the molecule. A net current of $0.22 \mathrm{nA}$ flows from source to drain at this stage. As the fermi level is further lowered due to rising positive bias, the current reduces due to receding overlap of fermi level and molecular orbitals. The reduction in current for increasing voltage exhibits negative resistance characteristic of the molecule. The current reduces till the fermi level is aligned with lower occupied MO and again there is display of positive resistance. For applied voltages from $2.7 \mathrm{~V}$ to $3.0 \mathrm{~V}$ a constant current of $0.17 \mathrm{nA}$ is maintained.This is due to closely located occupied MO's 31 , 32, 33 and 34. The fermi level glides along these MOs and current flows through them. The current again reduces till the fermi level is aligned with MO 30 and 29. This is implied by a discontinuity in the negative resistance characteristic for an applied voltage of $3.8 \mathrm{~V}$ and $3.9 \mathrm{~V}$ with a corresponding current of .039nA and .037nA respectively flowing through the structure. The current again reduces till it reaches a minimum value of $1.05 \times 10^{-5} \mathrm{nA}$ for an applied voltage of $4.5 \mathrm{~V}$. The current-voltage curve thus obtained is shown in Fig 6. For source voltages higher than $4.5 \mathrm{~V}$, there is rise in current again due to direct tunneling through the molecule. The curve plotted for the various current-voltage values is similar to that of a tunneling diode where $\mathrm{I}_{\text {peak }}$ is $0.22 \mathrm{nA}$ and $\mathrm{I}_{\text {valley }}$ is $1.05 \mathrm{X} 10^{-5} \mathrm{nA}$. The value of peak to valley ratio (PVR) for the adenine molecule with positive bias is calculated to be $2.1 \times 10^{4}$.

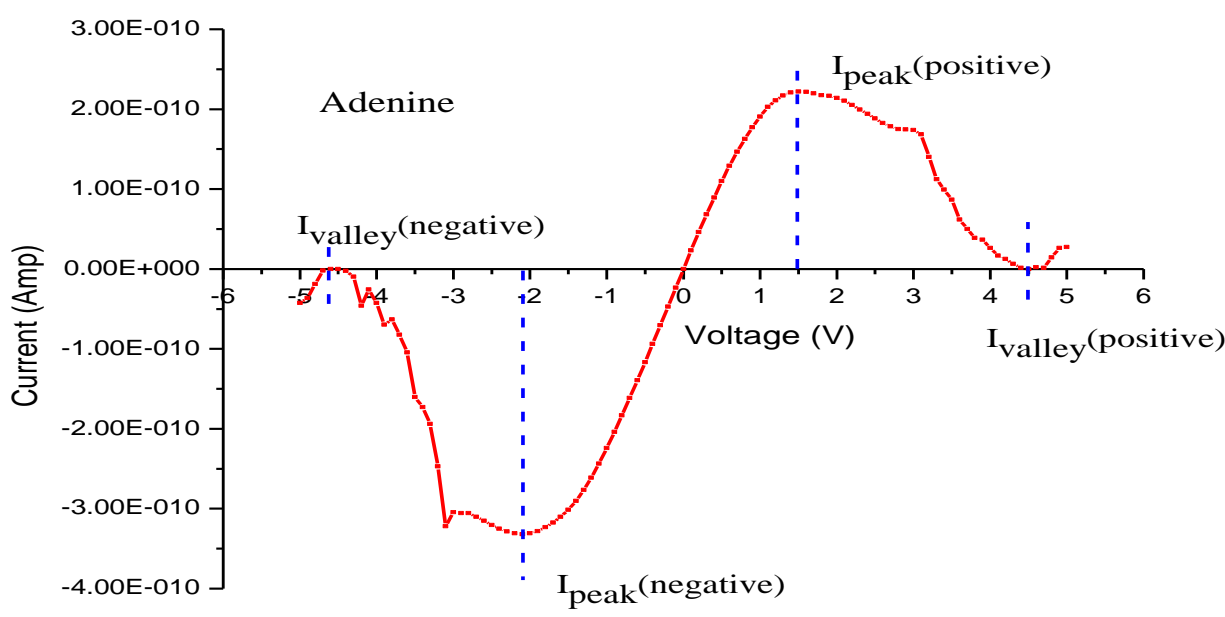

Fig 6 Current- voltage characteristics of DNA Base 'Adenine'

\subsubsection{Negative Source Voltage}

The magnitude of current increases in negative direction on application of negative bias till the Fermi level is aligned with LUMO (MO 36 with energy level $6.14 \mathrm{eV}$ ). This state is attained for a source voltage of $-2.1 \mathrm{~V}$, which produces a current of $0.33 \mathrm{nA}$ flowing from drain to source of the twoprobe setup displaying n-type of conduction. It is observed that molecule displays a positive resistance of $4.46 \times 10^{6} \mathrm{k} \Omega$. Further increase in magnitude of source potential takes the fermi level higher, sliding along MO's 37, 38, 39 and 40 and the inter-orbital gaps. It is observed that for voltages more negative than $-2.1 \mathrm{~V}$ the molecule displays negative resistance characteristic. Current peaks (as tabulated in Table 1) signifying resonant tunneling through the unoccupied molecular orbitals mark the negative resistance part of the curve.

The current peaks are distinctly visible as the unoccupied orbitals are more widely spaced as compared to the occupied orbitals. The reducing current reaches its maximum value of $2.76 \mathrm{X} 10^{-15} \mathrm{~A}$ at a source voltage of $-4.6 \mathrm{~V}$. The current again starts rising for higher values of negative applied potential due to direct tunneling. The molecule offers a PVR of $1.2 \times 10^{5}$ in this configuration.

Table 1 Resonant Current Peaks of Adenine

\begin{tabular}{|c|c|}
\hline Voltage (V) & Current Peaks (nA) \\
\hline-4.2 & $-4.59 \mathrm{E}-02$ \\
\hline-3.9 & $-6.97 \mathrm{E}-02$ \\
\hline-3.1 & $-3.22 \mathrm{E}-01$ \\
\hline-2.1 & $-3.32 \mathrm{E}-01$ \\
\hline 1.5 & $2.22 \mathrm{E}-01$ \\
\hline 1.6 & $2.22 \mathrm{E}-01$ \\
\hline
\end{tabular}

The various significant values observed from the currentvoltage characteristic are tabulated in Table 2 . It is noted that though PVR is higher for n-type of conduction both the 
configurations offer very large PVR, hence the Adenine molecule can be exploited for use in digital circuits.

Table 2 Peak to Valley Current Ratios for positive and negative bias

\begin{tabular}{|c|c|c|c|c|}
\hline $\begin{array}{c}\text { Peak } \\
\text { voltage } \\
\left(\mathbf{V}_{\text {peak }}\right)\end{array}$ & $\begin{array}{c}\text { Peak } \\
\text { Current } \\
\left(\mathbf{I}_{\text {peak }}\right)\end{array}$ & $\begin{array}{c}\text { Valley } \\
\text { Voltage } \\
\left(\mathbf{V}_{\text {valley }}\right)\end{array}$ & $\begin{array}{c}\text { Valley } \\
\text { Current } \\
\left(\mathbf{I}_{\text {valley }}\right)\end{array}$ & $\begin{array}{c}\text { Peak to } \\
\text { Valley } \\
\text { Current } \\
\text { Ratio } \\
(\text { PVR })\end{array}$ \\
\hline $1.5 \mathrm{~V}$ & $0.22 \mathrm{nA}$ & $4.5 \mathrm{~V}$ & $\begin{array}{c}1.05 \mathrm{X} \\
10^{-5} \mathrm{nA}\end{array}$ & $2.1 \times 10^{4}$ \\
\hline$-2.1 \mathrm{~V}$ & $-0.33 \mathrm{nA}$ & $-4.6 \mathrm{~V}$ & $-2.76 \mathrm{X}$ & $1.2 \times 10^{5}$ \\
& & & $10^{-6} \mathrm{nA}$ & \\
\hline
\end{tabular}

\subsection{Current -voltage characteristics of Guanine}

As in case of Adenine, a voltage bias varying from $-5 \mathrm{~V}$ to $+5 \mathrm{~V}$ with steps of $0.1 \mathrm{~V}$ was applied at the source of goldGuanine-gold structure with drain fixed at $0 \mathrm{~V}$. Current flow corresponding to the applied voltage are plotted to show the trend of current-voltage characteristics. The curve thus obtained is shown in Fig 8. The piecewise analysis was carried out for the three bias segments; zero bias, positive bias and negative bias.

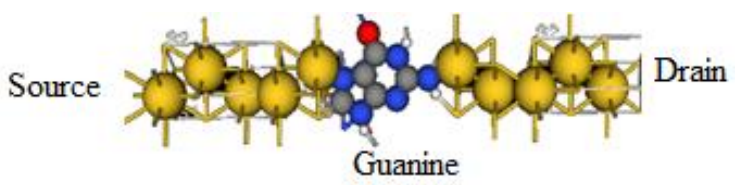

Fig 7 Gold-Guanine-Gold structure

\subsubsection{Zero source voltage}

Zero biasing corresponds to no-conduction condition. As the fermi energy level of the terminals lies in the middle of HOMO-LUMO gap $(12.62 \mathrm{eV})$ of the Guanine molecule. None of the molecular orbitals is aligned with the fermi energy level of terminals; hence there is no path for transmission of charge. The energy gap is much larger than room temperature thermal energy (thermal energy $=\mathrm{k}_{\mathrm{B}} \mathrm{T}=0.026 \mathrm{eV}$ at $\mathrm{T}=300 \mathrm{~K}$ ). This means that the molecule is thermally stable and the electronic charge distribution cannot be disturbed due to thermal effects. Hence the electrons are not able to move from source to molecule to drain or vice-versa resulting in zero current.

\subsubsection{Positive Source Voltage}

With increase in voltage, the current starts rising due to rise in transmission coefficients virtue the differential mismatch of the energy levels of source, molecule and drain. The initial resistance of $1.04 \times 10^{6} \mathrm{k} \Omega$ is offered by the molecule. For applied voltage of $1.4 \mathrm{~V}$, the fermi level of the source is aligned with the HOMO of the molecule that opens a conduction channel for flow of electrons from drain to source. Rising positive bias leads to reducing current due to receding overlap of fermi level and molecular orbitals. At this stage the molecule is displaying differential negative energy. The current reducing trend is followed till the fermi level is aligned with lower occupied MO and again there is enhanced conduction. A resonant peak is observed when current rises to value of $8.46 \mathrm{E}-$ $01 \mathrm{nA}$ is observed for an applied voltage of $2 \mathrm{~V}$. Two more resonant peaks are observed for source bias of $3.1 \mathrm{~V}$ and $3.9 \mathrm{~V}$ when current with value of $7.09 \mathrm{E}-01 \mathrm{nA}$ and $1.65 \mathrm{E}-01 \mathrm{nA}$ respectively. These resonance peaks appear as the fermi level glides down along the occupied MOs $38,37, \ldots$.and so on. The negative differential section ends when a valley current of 1.44E-05 nA flows for an applied voltage of 4.6V. For higher voltages the current starts rising again displaying typical tunnel diode characteristics. The Peak to Valley current ratio (PVR) of the Guanine molecule based resonant tunneling diode with positive source bias is $6.09 \times 10^{4}$.

\subsubsection{Negative Source Voltage}

Negative potential increases the electron population that results in elevation of the fermi energy level. Elevation pushes the fermi levels towards the unoccupied molecular orbitals. As it approaches the LUMO (MO 40) the conductance increases and the current rises till a current reaches its negative peak value of $-5.87 \mathrm{E}-01 \mathrm{nA}$ for an applied voltage of $-1.2 \mathrm{~V}$. When the voltage is increased the

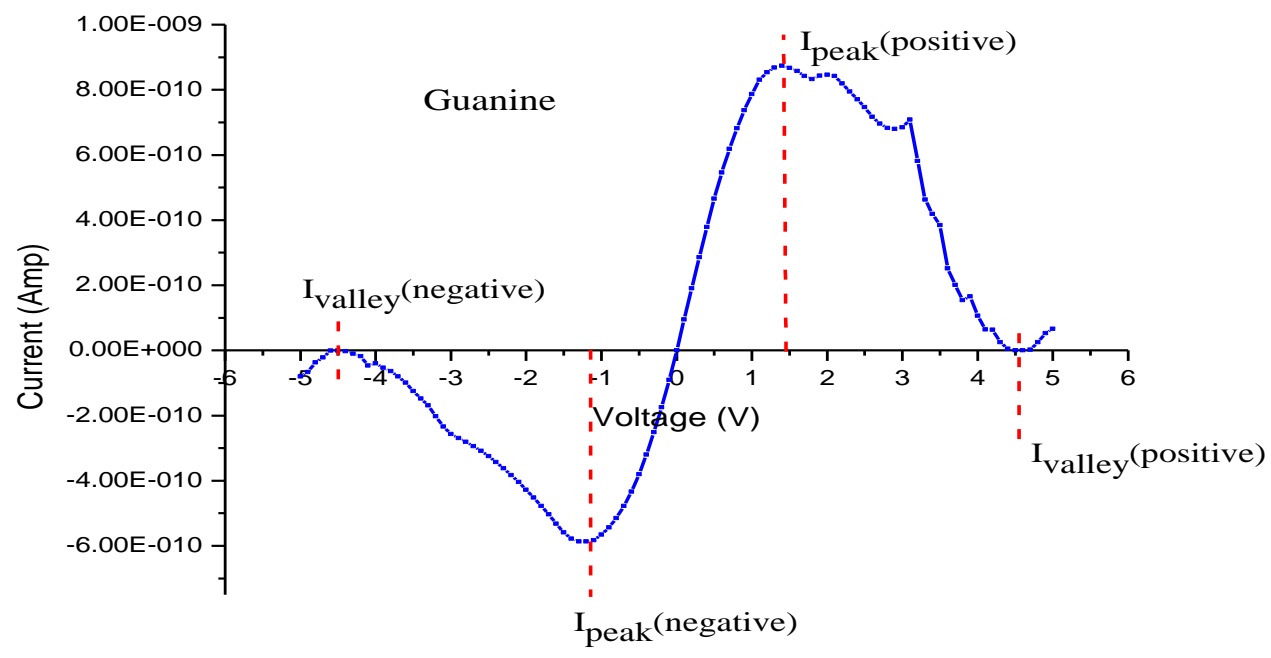

Fig 8 Current- voltage characteristics of DNA Base 'Guanine' 
current starts reducing, hence displaying negative resistance properties of the molecule. The differential negative resistance is marked by another resonant peak of $-4.65 \mathrm{E}-02 \mathrm{nA}$ when the source voltage $-4.2 \mathrm{~V}$ and it ends with a negative valley current of $-2.92 \mathrm{E}-04 \mathrm{nA}$ which is attained for a voltage of $-4.5 \mathrm{~V}$. The current profile again displays positive resistance for larger biases. The PVR in this case is given by the value $2.01 \times 10^{3}$.

Table 3. Resonant Current Peaks for Guanine

\begin{tabular}{|c|c|}
\hline Voltage (V) & Current (nA) \\
\hline-4.1 & $-4.65 \mathrm{E}-02$ \\
\hline-1.2 & $-5.87 \mathrm{E}-01$ \\
\hline 1.4 & $8.74 \mathrm{E}-01$ \\
\hline 2.0 & $8.46 \mathrm{E}-01$ \\
\hline 3.1 & $7.09 \mathrm{E}-01$ \\
\hline 3.9 & $1.65 \mathrm{E}-01$ \\
\hline
\end{tabular}

Table 4. Peak to Valley Current Ratios for Guanine

\begin{tabular}{|c|c|c|c|c|}
\hline $\begin{array}{c}\text { Peak } \\
\text { voltage } \\
\left(\mathbf{V}_{\text {peak }}\right)\end{array}$ & $\begin{array}{c}\text { Peak } \\
\text { Current } \\
\left(\mathbf{I}_{\text {peak }}\right)\end{array}$ & $\begin{array}{c}\text { Valley } \\
\text { Voltage } \\
\left(\mathbf{V}_{\text {valley }}\right)\end{array}$ & $\begin{array}{c}\text { Valley } \\
\text { Current } \\
\left(\mathbf{I}_{\text {valley }}\right)\end{array}$ & $\begin{array}{c}\text { Peak to } \\
\text { Valley } \\
\text { Current } \\
\text { Ratio } \\
(\mathbf{P V R})\end{array}$ \\
\hline $1.4 \mathrm{~V}$ & $0.87 \mathrm{nA}$ & $4.6 \mathrm{~V}$ & $\begin{array}{c}1.44 \mathrm{X} 10^{-} \\
5 \mathrm{nA}\end{array}$ & $\begin{array}{c}6.09 \mathrm{X} \\
10^{4}\end{array}$ \\
\hline$-1.2 \mathrm{~V}$ & $-0.58 \mathrm{nA}$ & $-4.5 \mathrm{~V}$ & $-2.92 \mathrm{X}$ & $2.01 \mathrm{X}$ \\
& & & $10^{-4} \mathrm{nA}$ & $10^{3}$ \\
\hline
\end{tabular}

\section{CONCLUSION}

Ab-initio study of the Adenine and Guanine molecules has been carried in this paper to study the conductivity properties. The current-voltage characteristics of both the molecules were obtained with an intention to predict the electronic behavior of the molecules which can be used for device modeling. Excellent tunneling currents flow through both the molecules. Resonant tunneling is also displayed in the negative differential resistance section of the characteristics. Both the factors suggest utilization of the molecules as the nanoelectronic devices called Resonant Tunneling Diodes. It is observed that PVR is very large for both the molecules. As similar behavior is observed in both the positive and negative bias domain, the molecules Adenine and Guanine can be exploited for use in designing positive and negative logic digital devices.

\section{REFERENCES}

[1] Schaller,R.R. 1997.Moore's Law: Past, Present And Future.

[2] Tour,J., Kozaki,M., and Seminario,J. M. 1998.Molecular Scale Electronics: A Synthetic/Computational Approach to Digital Computing.

[3] Chen, J., Reed, M. A., Rawlett, A. M., and Tour, J. M.1999. Observation of a Large On-Off Ratio and Negative Differential Resistance in an Electronic Molecular Switch

[4] Tsutsui,M.,Taniguchi,M.,Yokota,K., and Kawai,T.2010. Identifying single nucleotides by tunnelling current.

[5] Sun, J.P., Haddad, G.I., Mazumder, P.,and Schulman, J.N.1998. Resonant tunneling diodes: Models and properties.

[6] Bjork, M.T., Ohlsson, B.J., Thelander, C.et al. 2002. Nanowire resonant tunneling diodes.

[7] Mayer,T.S.,Cai, L.T.,Yoon, H. et al. Bistable switching in nanoscale molecular junctions.

[8] Seminario,J.M., Zacarias,A.G. and Tour,J.M.2000. Theoretical study of a molecular resonant tunneling diode.

[9] Keane, Z.K., Ciszek, J.W.,Tour,J.M. and Natelson, D.2006. Three-terminal devices to examine singlemolecule conductance switching.

[10] Sun,J.P., Haddad,G.I., Mazumder,P. and Schulman,J.N., 1998.Resonant Tunneling Diodes: Models and Properties. 